\title{
RELATION BETWEEN LASER FLUX, TEMPERATURE AND \\ IONISATION EQUILIBRIUM IN LASER PRODUCED PLASMAS
}

\author{
M. H. KEY, R. J. HUTCHEON, D. A. PRESTON, and T. P. DONALDSON \\ The Queen's University, Belfast, Northern Ireland
}

\begin{abstract}
Laser produced plasmas are a useful source of highly stripped ions for XUV spectroscopy and it is therefore important to understand the relation between the incident laser flux, plasma temperature and ionisation equilibrium.

An experiment has been carried out in which a $2 \mathrm{GW}, 15 \mathrm{nS}$ pulse of neodymium laser radiation, of known power, was focussed on a solid target. The flux density at the target was obtained from photographic measurements on a highly magnified image of a duplicate focal spot formed by a beam splitter and identical lens. The plasma temperature was obtained from foil absorption measurements and the highest stage of ionisation from the XUV spectrum.

The measured plasma temperatures are plotted against the laser power on Figure 1. These points can be represented by the relation temperature $\propto$ (power) ${ }^{x}$ where $0.66 \leqslant x \leqslant 0.75$. Some of these points are plotted in terms of laser flux and compared with the theoretical models of Fauquignon and Floux (1970) and Puell (1970) on Figure 2. The closest agreement is with the Fauquignon model. The wide error bars correspond to the uncertainty in the focal flux distribution. (The flux $\phi_{c}$ mentioned
\end{abstract}

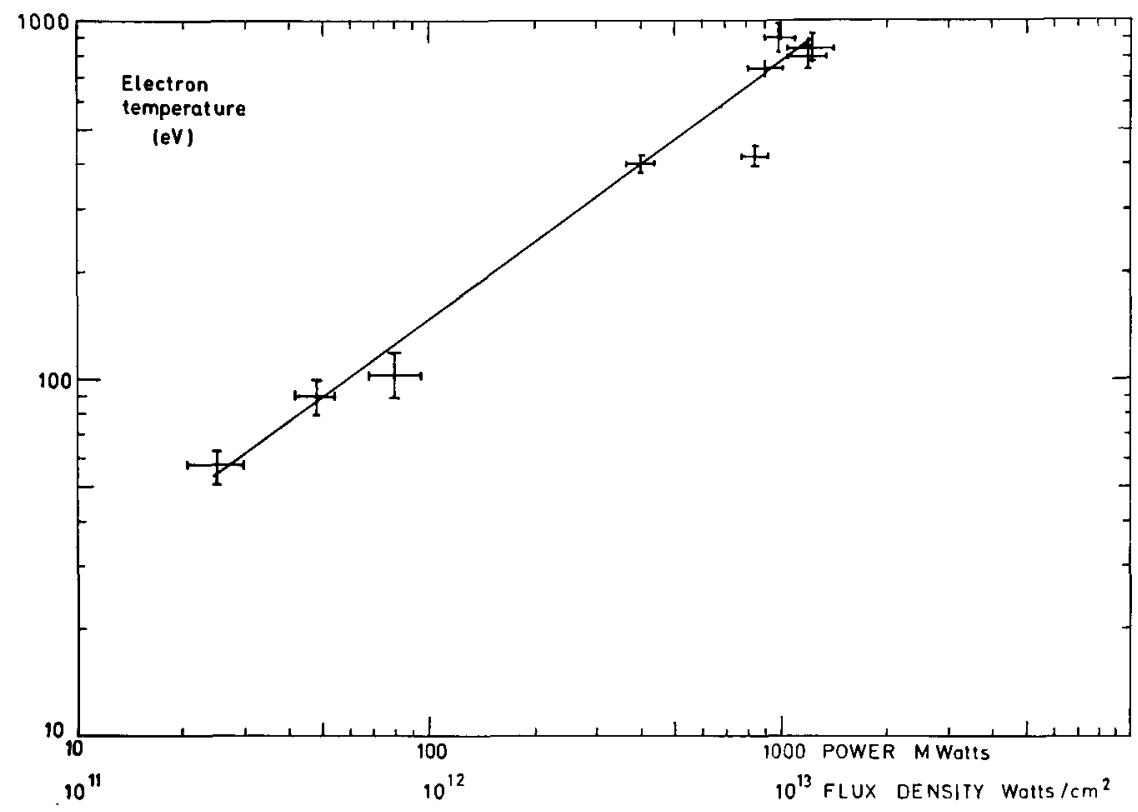

Fig. 1. Electron temperature as function of laser power (flux density values approximate). 


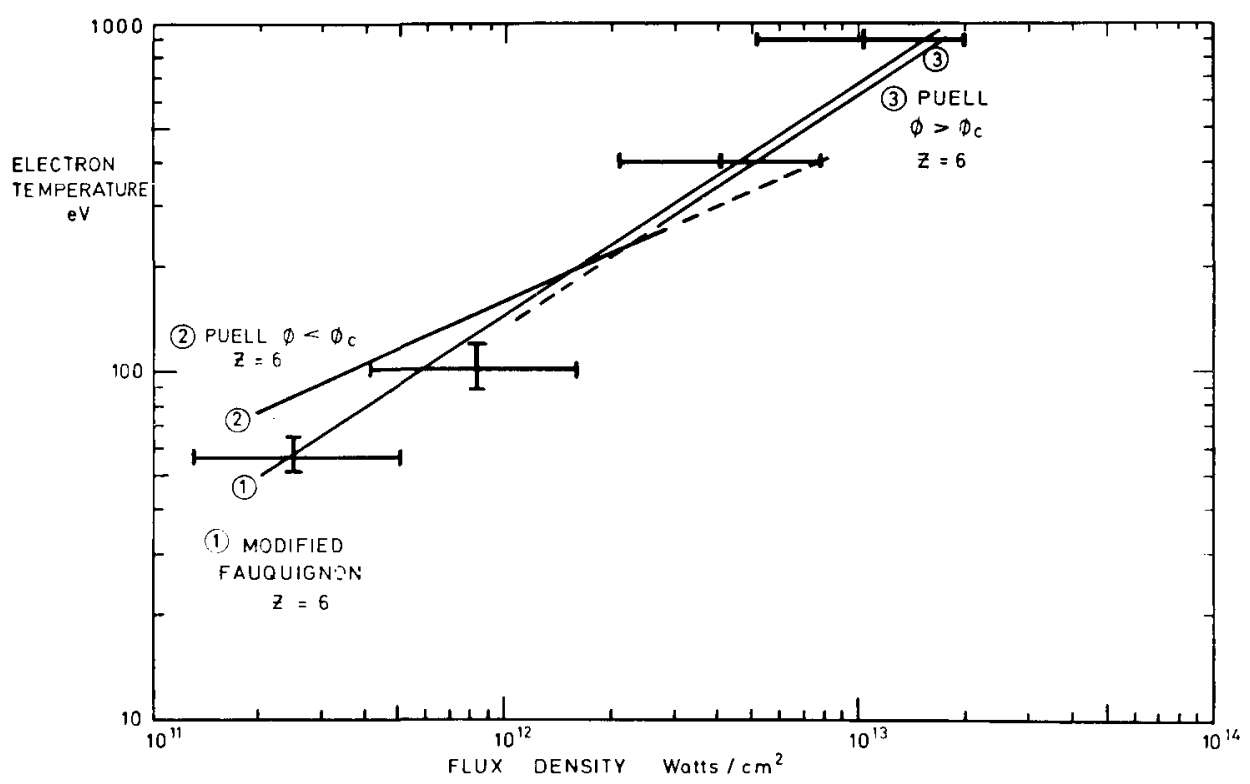

Fig. 2. Electron temperature as function of laser flux density.

on this graph is a critical flux density which occurs in the Puell theory.) A thick plane surface perspex target was generally used, although other carbon based targets, e.g. P.T.F.E. were also used giving no measurably different temperature for a given flux density. The theoretical predictions of the Fauquignon model, are labelled 'modified' on Figure 2, because this model was originally described for a solid deuterium target and the theoretical calculations required adjustment to allow for the change in the average atomic number of the target material.

Knowledge of the dependence of plasma temperature on incident laser flux should lead to estimates of the degree of ionisation obtained. However, such estimates should allow for the possibility of ions leaving the dense plasma region before the ionisation equilibrium appropriate to the plasma temperature is reached. Theoretical estimates suggest that, for the laser conditions used, this effect might influence the ionisation equilibrium of hydrogenic ions with $Z=8$ or higher. However, the experimentally determined threshold temperature for observation of both $\mathrm{O}$ vIII and F IX ions is $280 \pm 70 \mathrm{eV}$. This temperature agrees with that required in a steady state plasma.

\section{References}

Fauquignon, C. and Floux, F.: 1970, Phys. Fluids, 13, 386.

Puell, H.: 1970, Z. Naturforsch 25a, 1807. 